DOI: $10.1002 / s m l l .200701236$

\section{Embedded Nanofibers Induced by High-Energy Ion Irradiation of Bulk GaSb**}

\author{
Alejandro Perez-Bergquist, Sha Zhu, Kai Sun, Xia Xiang, \\ Yanwen Zhang, and Lumin Wang*
}

Semiconductor nanostructures have been attracting widespread attention for their unique quantum-confined nanoscale properties. In particular, the luminescence properties of nanoscale semiconductors are seen as a key to the future of optoelectronic microdevice fabrication. One material that has received substantial attention in the field is GaSb, a direct-bandgap semiconductor that has been used to fabricate high-frequency, low-power-consumption electronic devices, ${ }^{[1]}$ optoelectronic devices with varying wavelengths, ${ }^{[2]}$ and ordered semiconductor quantum dots. ${ }^{[3]}$ Previous studies using ion accelerators $^{[4]}$ and more recently using focused ion beams (FIBs) ${ }^{[5-7]}$ have shown that ion irradiation of GaSb under appropriate implantation conditions results in the formation of porous surface structures, similar to those observed previously in ionirradiated Ge. ${ }^{[-10]}$ Depending on the ion energy and fluence, either porous cellular structures or nanofiber layers can result. Nitta and Taniwaki proposed a formation mechanism for the $\mathrm{GaSb}$ porous layer based on the formation and diffusion of point defects induced by ion irradiation, ${ }^{[11-14]}$ and subsequent studies have added to the growth model in an attempt to explain observed behavior of the porous layer. ${ }^{[6,7]}$

Although numerous techniques exist for the production and modification of various nanostructures on material surfaces, fabrication of such structures embedded underneath the material surface have proved elusive. In this Communication, we present the novel formation of embedded nanofiber layers under the intact surface of GaSb single crystals by high-energy $\mathrm{Au}^{+}$ion irradiation, and we analyze the distinct regions of the fiber layers formed as a result. Using the newly obtained data, previous growth models are examined and a new universal

[*] A. Perez-Bergquist, Dr. S. Zhu, Dr. K. Sun, X. Xiang, Prof. L. M. Wang Department of Nuclear Engineering and Radiological Sciences Department of Materials Science and Engineering University of Michigan

2355 Bonisteel Blvd., Ann Arbor, MI 48109-2104 (USA)

Fax: $(+1)$ 734-647-8531

E-mail: Imwang@umich.edu

Dr. Y. W. Zhang

Pacific Northwest National Laboratory (PNNL)

P.O. Box 999, Richland, WA 99352 (USA)

[**] This study was supported by the U.S. DOE (DE-FG02-02ER46005 and DE-AC05-76RL01830). Ion implantations were conducted in the Environmental Molecular Sciences Laboratory at PNNL and analyses were completed at the University of Michigan's Electron Microbeam Analysis Laboratory using equipment supported in part by the NSF (DMR-0320740 and DMR-9871177). model is developed to account for results under a broad spectrum of experimental conditions.

The novel embedded fiber layers were formed by irradiating a stationary $\mathrm{GaSb}$ sample with $1 \mathrm{MeV} \mathrm{Au}^{+}$ions at perpendicular incidence to ion fluences of $1 \times 10^{14}{\text { ions } \mathrm{cm}^{-2}}^{-2}$ to $6 \times 10^{15}$ ions $\mathrm{cm}^{-2}$. After ion irradiation to $1 \times 10^{14}$ ions $\mathrm{cm}^{-2}$, three distinct regimes were visible in the GaSb sample cross section by scanning electron microscopy (SEM), as shown in Figure 1. At the sample surface, the sample shows a thin intact surface layer of uniform thickness (the top portion of Figure 1b). At this ion fluence, the thickness of the surface layer was measured to be roughly $20 \mathrm{~nm}$. At higher fluences of up to $6 \times 10^{14}$ ions $\mathrm{cm}^{-2}$, the surface layer remained uniform in thickness, at $20 \pm 3 \mathrm{~nm}$ as measured by SEM. At ion fluences higher than $6 \times 10^{14}$ ions $\mathrm{cm}^{-2}$, the surface layer tends to be removed, primarily due to mechanical stress from volume expansion of the subsurface fiber layer. At ion fluences greater than $1 \times 10^{15}$ ions $\mathrm{cm}^{-2}$, the GaSb fibers became completely exposed, with a cobweblike structure beginning to speckle the surface of the irradiated area above $6 \times 10^{15}$ ions $\mathrm{cm}^{-2}$.

Directly underneath the thin surface layer is a regime of fully formed, relatively uniformly spaced GaSb nanofibers of $\approx 20 \mathrm{~nm}$ in diameter. At a fluence of $1 \times 10^{14}$ ions $\mathrm{cm}^{-2}$, the fiber layer measured roughly $1.95 \mu \mathrm{m}$ in thickness (Figure 1a), and the layer continued to increase in thickness with increasing ion fluence. The fiber layer retained its structural integrity up through a fluence of $6 \times 10^{14}$ ions $\mathrm{cm}^{-2}$, at which point the layer measured $6 \mu \mathrm{m}$ in thickness. However, at higher ion fluences the fiber layer became increasingly fragile and the thickness was no longer uniform. At the maximum tested ion fluence of $6 \times 10^{15}$ ions $\mathrm{cm}^{-2}$, portions of the GaSb fiber layer reached a thickness of roughly $20 \mu \mathrm{m}$. The fiber diameter, which was measured to be in the range of $20 \pm 4 \mathrm{~nm}$ from electron microscopy images, remained fairly uniform both within each sample and across the range of ion fluences (Figure 2). Increasing the ion fluence from $1 \times 10^{14}$ to $6 \times 10^{15}$ ions $\mathrm{cm}^{-2}$ did not appear to affect the average fiber diameter (Figure 2a). In addition, separate GaSb samples were irradiated with $150 \mathrm{keV} \mathrm{Kr}{ }^{+}$ions to a variety of ion fluences. $\mathrm{Au}^{+}$and $\mathrm{Kr}^{+}$ ions were used to avoid reaction with the material whilst also allowing observation of changes due to both metallic and gaseous ion implantation over a variety of ion energies. The $\mathrm{Kr}^{+}$implanted samples also resulted in very uniform fibers of $\approx 16 \mathrm{~nm}$ in diameter (Figure $2 \mathrm{~b}$ ). Seemingly, GaSb nanofiber diameter is only weakly affected by ion mass, energy, and fluence, as evidenced by our experimentation and values reported in the literature. ${ }^{[6,11,14]}$ It appears that increased ion fluence mildly decreases fiber size, while use of heavier implantation ions results in slightly larger fibers, but an in depth study of fiber size as a function of implantation parameters is needed before definitive trends can be extrapolated. Transmission electron microscopy (TEM) measurements indicate the presence of nanocrystals within the GaSb fibers, as also reported in the literature ${ }^{[7]}$ but the majority of the fiber volume remains amorphous (Figure 2c).

At the bottom of the sample cross section, a transitional nanoporous layer contains voids $10 \mathrm{~nm}$ in diameter (Figure $1 \mathrm{~d}$ ), as compared to $\approx 50 \mathrm{~nm}$ in diameter in the fiber regime. This transitional layer is a formation regime, in which the bulk of the 


\section{communications}
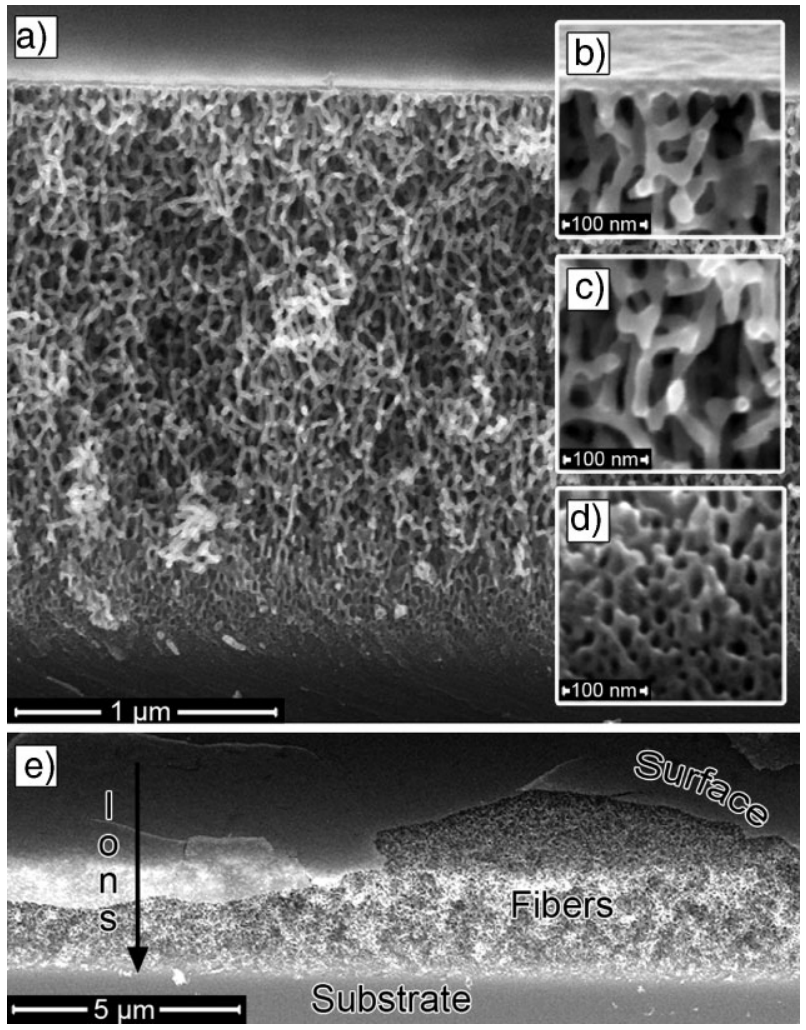

Figure 1. a) Cross-sectional SEM image of GaSb fiber layers irradiated with $1 \mathrm{MeV} \mathrm{Au}^{+}$to $1 \times 10^{14}$ ions $\mathrm{cm}^{-2}$. Insets show $\mathrm{b}$ ) the surface layer (tilted to $10^{\circ}$ off axis), c) the presence of fully formed fibers, and d) a transition regime in which the fibers blend into the substrate. e) The same sample is shown at an angled perspective, showing the incident ion beam direction, surface layer, fiber layer, and crystalline substrate.

incident ion energy is deposited. As the incident $\mathrm{Au}^{+}$ions slow down, they rapidly begin to lose energy through atomic collision cascades, resulting in a thin porous layer and a quick transition from $\mathrm{GaSb}$ fibers to the dense substrate. A thin layer of partially amorphous $\mathrm{GaSb}$, caused by limited atomic collision damage, ${ }^{[10]}$ separates the porous region from the crystalline substrate. ${ }^{[12]}$
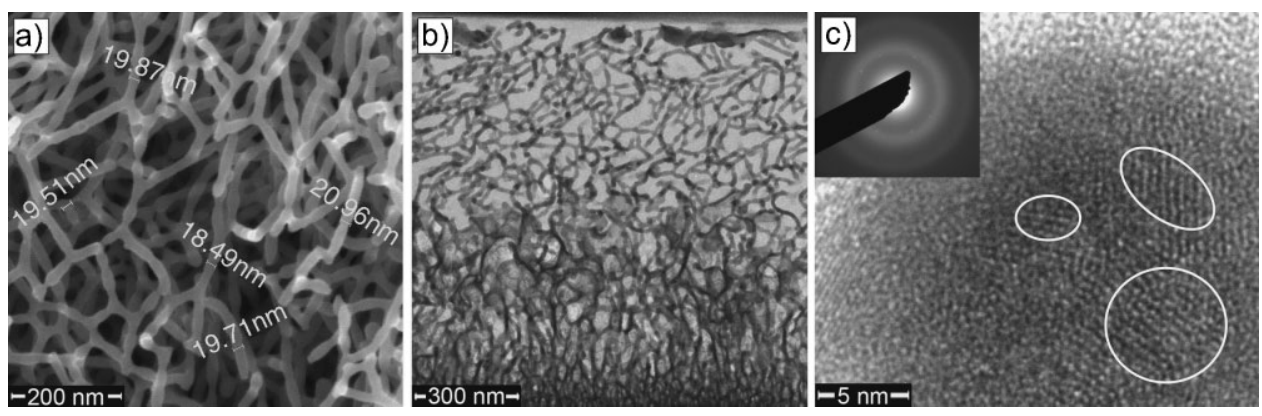

Figure 2. a) GaSb fibers irradiated at $6 \times 10^{14}$ ions $\mathrm{cm}^{-2}$ with $1 \mathrm{MeV} \mathrm{Au}^{+}$ions. SEM imaging shows that fiber diameter is fairly uniform and does not change at increased ion fluence. b) GaSb fibers irradiated at $6 \times 10^{15}$ ions $\mathrm{cm}^{-2}$ with $150 \mathrm{keV} \mathrm{Kr}^{+}$ions. Cross-sectional TEM image shows that fiber size also remains fairly constant at varying implantation energies. c) HRTEM of a single fiber reveals that multiple nanocrystals are present within the fiber, and diffraction of the fiber volume (inset) shows distinct halos, confirming the presence of nanocrystals in the bulk fiber mass.
At the sample surface, after an ion fluence of $1 \times 10^{14}$ ions $\mathrm{cm}^{-2}$, the entire irradiated area of the sample was covered by a uniform surface layer, but as ion fluence increased, the surface layer was progressively removed in a nonlinear fashion. Initially, only very small breaks resembling polymer crazing occur in the surface layer (Figure 3a). As the ion fluence increases, the breach in the surface layer expands outwards, exposing increasingly greater amounts of the embedded nanofibers (Figure 3b). When two openings in the surface layer expand into one another, the expansion fronts are pulled into thin strands (Figure 3c). As ions continue to bombard the surface, the surface layer begins to detach from the fiber layer, with large patches of the surface beginning to crack and spall off (Figure 3d). Eventually, the entire surface is removed but further ion irradiation creates cobweb-like structures uniformly distributed across the exposed fiber surface (Figures $3 e$ and f). Tests performed with low-energy and high-energy ions all resulted in the formation of web-like surface structures at high ion fluence, showing that these structures are not related to the presence or removal of a surface layer over the fibers. Highfluence irradiation experiments demonstrate that the formation of the web structures is a side effect of ion-damage degradation of the fibers.

As showcased in Figure 3a, openings in the surface layer initially tend to be long and thin and they often are arranged in parallel orientations. However, investigation of the break orientations shows no crystallographic dependence, indicating that some other formation mechanism is responsible. As the breaks expand, they tend to retain their initial orientation until, with increasing ion fluence, surface openings begin to intersect and overlap, causing all semblance of an ordered removal method to be lost.

A defect structure formation mechanism has been developed in detail by Nitta et al. that describes the formation process of porous $\mathrm{GaSb}$ at relatively low ion-implantation energies $(\approx 60 \mathrm{keV}) .{ }^{[1-14]}$ To confirm the current model for low-energy ions, we performed in situ SEM and ion irradiation with $30 \mathrm{keV} \mathrm{Ga}^{+}$ions in a traditional Ga-based dual-beam FIB SEM system. The results from the in situ experiments, shown in Figure 4, effectively confirm the model. At first, low-energy ions create a thin porous layer directly beneath the surface (Figure 4b). As ion fluence increases, the surface layer is breached, exposing the thin porous region (Figure $4 \mathrm{c}-\mathrm{e}$ ). Eventually, at an even higher ion fluence, the porous structures grow outwards, developing into a well-defined area of surface nanofibers (Figure 4f). However, this formation mechanism breaks down for ion implantation at higher energies, in this case with $1 \mathrm{MeV} \mathrm{Au}^{+}$, as evidenced by the presence of a thick, embedded-fiber region as seen in Figure 1. A formation mechanism for higher-energy ions is shown in Figure 5. 


\section{small}
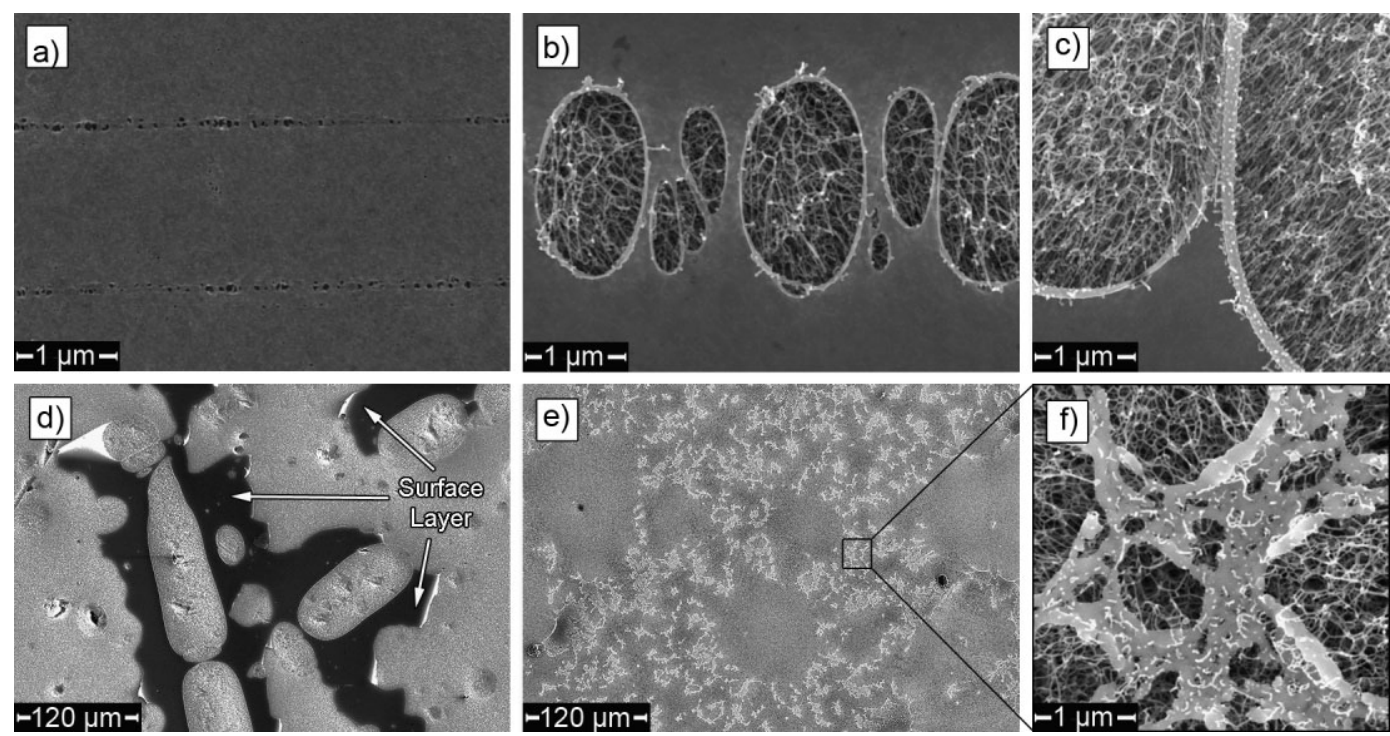

Figure 3. GaSb surface response to varying ion fluence. a) The GaSb surface layer is first breached with $1 \mathrm{MeV} \mathrm{Au}^{+}$ions at a fluence of around $1 \times 10^{14}$ ions $\mathrm{cm}^{-2}$. b) As the ion fluence increases, the surface layer is stretched, exposing the embedded nanofibers. c) Continued stress further increases the size of the openings and pulls the surface into taffy-like strands. d) Eventually, the majority of the surface layer begins to crack and peel away, exposing the fibers to the surface. e, f) Continued ion irradiation past this point results in degradation of the fibers, evidenced by small web-like patches speckling the surface.

At first, incident ion irradiation results in the formation of point defects and some surface sputtering. Because of highly inefficient recombination of vacancies and interstitials in $\mathrm{GaSb},{ }^{[15]}$ vacancies preferentially coalesce into voids that continue to grow until vacancy diffusion in the remaining nanofibers allows voids to annihilate at fiber walls. ${ }^{[6]}$ The same diffusion mechanism is active in the surface layer, which explains why the diameter of the fibers and the thickness of the surface layer are roughly equal. As voids accumulate, the density of the material drops dramatically, allowing incident ions to penetrate deeper into the substrate. Due to the nature of high-energy ion bombardment, the majority of atomic collisions happen at the end of the ion range, where ion energy is relatively low and nuclear stopping is predominant. Thus,
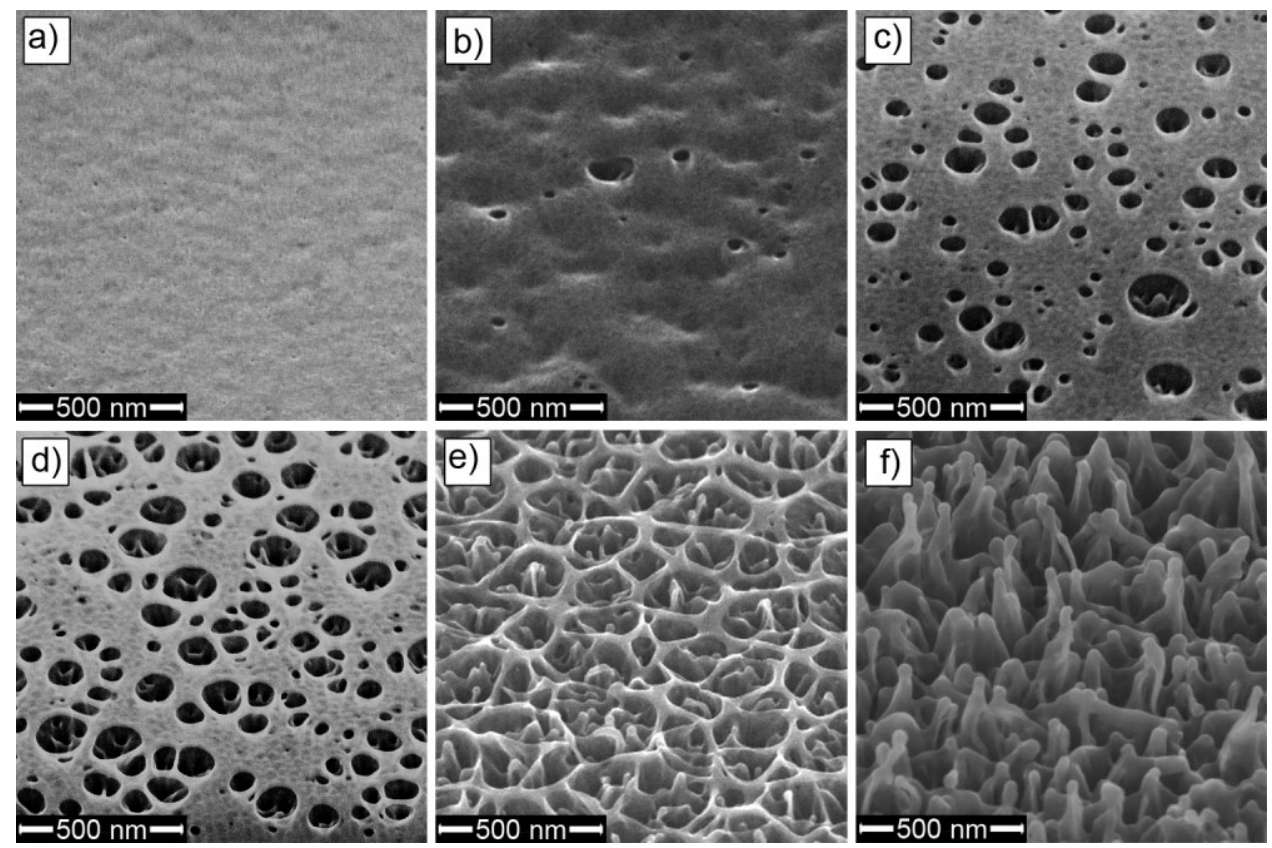

Figure 4. In situ SEM observation of GaSb surface under FIB irradiation with $30 \mathrm{keV} \mathrm{Ga}^{+}$ions. Irradiation fluence is: a) unirradiated, b) $1 \times 10^{15}$ ions $\mathrm{cm}^{-2}$, c) $1.3 \times 10^{15}$ ions $\mathrm{cm}^{-2}$, d) $1.6 \times 10^{15}$ ions $\mathrm{cm}^{-2}$, e) $2.6 \times 10^{15}$ ions $\mathrm{cm}^{-2}$, f) $6.5 \times 10^{15}$ ions $\mathrm{cm}^{-2}$. Surface morphology supports the current model for low-energy ion irradiation, showing the initial formation of porosity just underneath the surface followed by surface exposure and fiber formation. Samples are tilted to $52^{\circ}$. incident ions continue to cause void formation and growth at the bottom of the fiber layer (at the interface with the substrate) while resulting in less damage to the region directly underneath the surface layer and minimal damage to the surface layer itself. As a result, surface sputtering is all but eliminated, explaining why the surface-layer thickness remains constant at increasing ion fluence. Essentially, once the porous layer is formed, atomic collisions in the low-density porous layer are minimized, which helps to explain the relative uniformity in fiber diameter at increasing ion fluences, although diffusion mechanics and surface-energy considerations could also be important factors controlling fiber size.

Overall, irradiation with high-energy ions causes 


\section{communications}
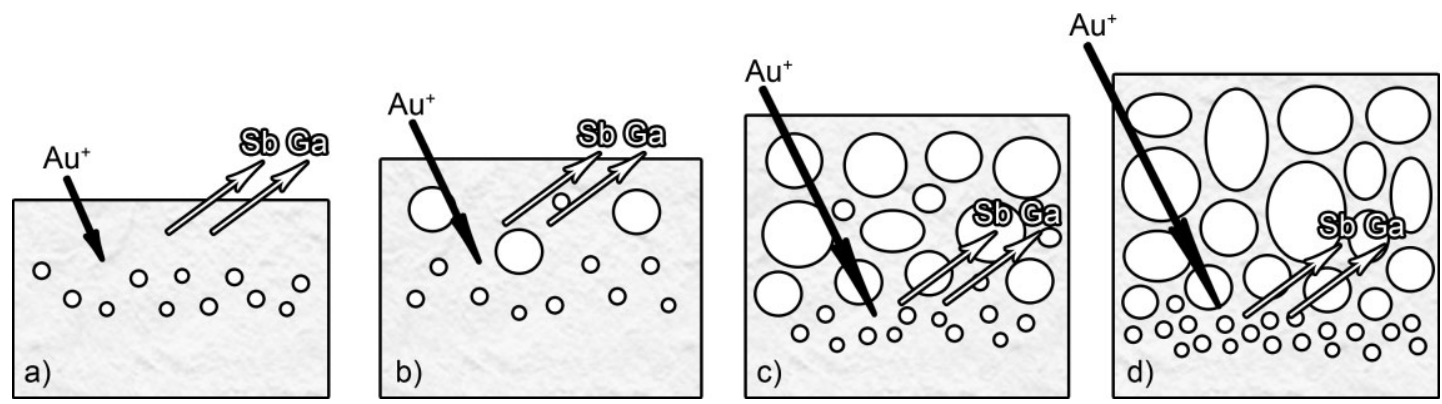

Figure 5. Nanofiber formation mechanism for higher-energy ions, with a two-dimensional GaSb cross section shown under ion irradiation. a) Incoming ions cause the formation of vacancies and b) as vacancies agglomerate, voids form. c) As voids accumulate, the material becomes less dense, allowing further penetration by the incoming ions, so much so that d) incoming ions are virtually unaffected by the void layer, and surface sputtering is all but eliminated.

continuous void growth at the bottom of the fiber layer, where nuclear stopping is significant. Because the energy of the incoming ion is weakly curtailed by the sparse fibers, the incoming ion continually deposits the bulk of its energy through atomic collisions in the dense transition regime. This model of continuous fiber growth upon ion irradiation breaks down at very high fluences, when the fiber layer becomes thick enough that significant atomic energy losses begin to take place in the fiber layer rather than in the substrate. With $1 \mathrm{MeV} \mathrm{Au}{ }^{+}$ions, this occurred at about $1 \times 10^{15}$ ions cm$^{-2}$ in a fiber layer thickness of roughly $13-15 \mu \mathrm{m}$. Despite fiberdegradation effects being visible in fiber layers of $15 \mu \mathrm{m}$ in thickness, fiber layers of up to $20 \mu \mathrm{m}$ were visible in some highfluence samples, as mentioned earlier. Statistical variation of ion ranges alone cannot explain this increased fiber layer growth at high ion fluence, but $\mathrm{Ga}$ and $\mathrm{Sb}$ vacancy diffusion could be responsible for continued fiber growth. $\mathrm{Ga}$ and $\mathrm{Sb}$ diffuse at different rates in $\mathrm{GaSb},{ }^{[16]}$ and vacancy in-diffusion into the GaSb substrate is a possible method by which fibers could continue to form past the projected ion range.

Investigation of $\mathrm{GaSb}$ samples irradiated with 2 and $3 \mathrm{MeV}$ $\mathrm{Au}^{+}$ions showed that a surface layer of roughly the same thickness $(\approx 20 \mathrm{~nm})$ was also formed, albeit at slightly higher incident ion fluence. Since high-energy ions initially slow down primarily through electronic and not atomic collisions, higherenergy ions produce more atomic vacancies deeper within the material and fewer vacancies near the surface region. As a result, a higher ion fluence is required for the surface layer to accumulate sufficient vacancies to reach the "fully formed" stage.

Ultimately, the GaSb porous structure is dependent upon the ion damage imparted to the material or, more specifically, the number of vacancies produced. Using the transport and range of ions in matter (TRIM) code,${ }^{[17]}$ vacancy production per ion was calculated for the surface regime under irradiation by 1,2 , and $3 \mathrm{MeV} \mathrm{Au}{ }^{+}$ions. Combining the results from the TRIM calculations with experimental data of the fiber structure as a function of ion fluence, a critical vacancy concentration was found at which both surface layer and fibers are fully formed. Results indicate that when the local vacancy concentration reaches between 1.5 and $2 \times 10^{22}$ vacancies $\mathrm{cm}^{-3}$ (a dose of $\approx 1$ displacement per atom, or dpa), the GaSb fibers reach a "fully formed" stable size and are not strongly affected by further ion bombardment. TRIM data indicates that ultrahigh-energy ions at relatively low fluence should be able to produce fully formed fibers embedded fairly deeply within a sample, and ultrahigh-energy-ion tests in germanium have shown that embedded porosity is possible.$^{[18]}$ Experimental

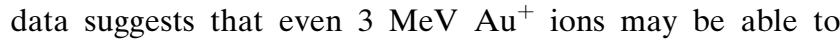
produce a void-free surface layer thicker than $\approx 20 \mathrm{~nm}$, albeit at very low fluences of $1 \times 10^{13}$ ions cm $\mathrm{cm}^{-2}$ or less but further experimentation is needed to confirm the mechanics of fiber growth at high energy and low fluence.

Consequently, the presence of a surface layer is directly related to ion-implantation energy. Ion irradiation of $\mathrm{GaSb}$ with low-energy ions does not appear to be capable of producing a thick, embedded fiber layer because irradiation with low-energy ions causes surface sputtering that rapidly removes the surface film. Sputtering effects are much less significant with higher-energy ions, as surface-sputtering yields drop rapidly due to expansion of the fiber layer with increasing fluence, yet the surface layer is still removed by increasingly higher ion fluences. Figure 6 presents a surface-layer-removal mechanism for high-energy ion bombardment of GaSb.

The key to the removal of the surface film seems to lie in the volume swelling of the fiber layer. At low ion dose ( $\approx 1 \mathrm{dpa}$ ), little swelling has occurred, leaving the surface layer under stress but still intact. As the underlying fiber layer continues to swell, it places the surface layer under tension, causing breaks in the surface to appear. Uniform stresses should result in breaks that are more or less aligned with one another, as in Figure 3a, but localized stress concentrations due to uneven swelling of the fiber layer can cause random alignments of breaks. At high dose ( $\approx 15 \mathrm{dpa})$, massive swelling causes breaks in the surface to crisscross and overlap. The bottom of the surface layer, previously under compressive stress from volume expansion, curls upwards when released from the underlying fibers; curling of the surface layer results in cracking and flaking off of the remaining patches of surface film.

In general, removal of the surface layer is a function of swelling of the underlying fiber layer and is a purely mechanical removal mechanism. Since swelling due to ion irradiation is directly related to vacancy concentration, the 


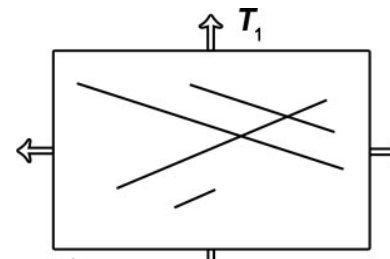

a)

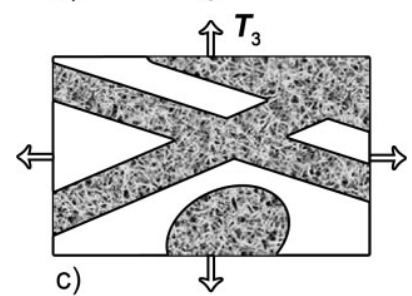

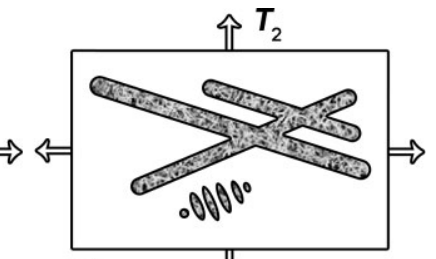

b)

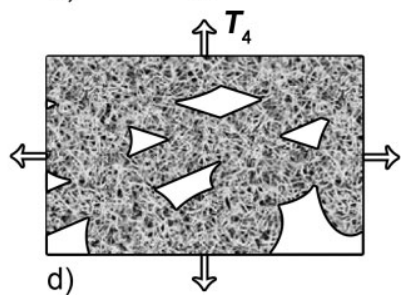

d)
Figure 6. Surface-layer-removal mechanism for GaSb is shown in a topdown view, with tensile force increasing from $T_{1}$ to $T_{4}$. a) Initially, little swelling has occurred, leaving the surface intact, although very slight defects can be seen at high magnification. b) As the underlying fibers swell, the surface layer is placed in tension, causing plastic deformation and exposing the fibers. c) With increased swelling, breaks expand, until d) at high fluence, massive swelling results in uneven stress between the top and bottom of the surface layer, causing the surface film to crack and peel off.

overall swelling and surface response is a function of the atomic damage imparted to the material. By controlling the incident ion fluence, energy, and mass, an embedded fiber layer can be formed where the surface swelling and removal effects are mitigated to the best degree possible.

Validity of this removal mechanism hinges on the plasticity of amorphous $\mathrm{GaSb}$ at the nanoscale. Bulk GaSb is brittle but the nanoplasticity of amorphous semiconductors is not well understood. Recent evidence seems to support the idea that nanoscale semiconductors may deform plastically, ${ }^{[19,20]}$ especially while under ion irradiation, ${ }^{[21]}$ and our visual evidence of the GaSb surface layer seems to support these claims. The craze-like openings in Figure $3 \mathrm{a}$ and the taffy-like strands seen in Figure $3 c$ are consistent with characteristics of a material undergoing plastic deformation.

Using the available growth models and our data, we can quantify the effects of the various implantation parameters that produce $\mathrm{GaSb}$ fiber layers. First, the thickness of the fiber layer can be tailored by adjusting the incident ion fluence. An increase in ion fluence will increase the thickness of the fiber layer until fiber-degradation mechanisms take over at very high ion fluence. The incident ion mass and energy determine at what depth in the GaSb sample the majority of atomic displacement damage occurs. By changing the ion mass and energy, one should be able to dictate the presence and thickness of a surface layer. Heavy, low-energy ions create purely surface structures, while light, very high energy ions are capable of producing embedded fibers, as evidenced in $185 \mathrm{MeV} \mathrm{I}^{+}$irradiated Ge. ${ }^{[18]}$ Two mechanisms affect the response of the surface layer, sputtering and volume expansion of the fiber layer. With low-energy ions, sputtering is significant and the surface cover is rapidly removed. With higher ion energies, where sputtering is not significant, the surface layer is stable to higher ion fluences but eventually the surface layer is removed by the expansion of the embedded fiber layer. Also, it is essential to mention temperature as another important parameter that strongly affects the growth of the fiber layer by controlling the mobility of vacancies and interstitials in the matrix. Although not covered in this Communication, ion implantation at either considerably low or high temperatures tends to inhibit void and fiber growth, as reported in the literature. ${ }^{[6]}$

In summary, high-energy ion irradiation was used to fabricate novel, distinct embedded layers of nanofibers ( $\approx 20 \mathrm{~nm}$ in diameter) within bulk GaSb, and the thickness of the fiber layer can be tailored by adjusting the incident ion energy and fluence. The ability to produce embedded fiber layers of specific thicknesses in GaSb may well have vast applications in the fabrication of microelectromechanical systems (MEMS) and other microelectronic and optoelectronic devices. The results also suggest that by using higherenergy ions at lower ion fluences, the thickness of the void-free surface layer can also be modifiable, allowing deep, subsurface fiber formation. Observation of the behavior of GaSb surface films under irradiation lends credence to the idea that amorphous semiconductors may behave plastically at the nanoscale.

\section{Experimental Section}

Mirror-polished (100) single-crystal GaSb wafers were irradiated at room temperature with $1-3 \mathrm{MeV} \mathrm{Au}^{+}$ions using a National Electrostatics Corporation 9SDH-2 3.0-MV tandem electrostatic accelerator at PNNL to fluences ranging from $3.9 \times 10^{13}$ ions $\mathrm{cm}^{-2}$ up to a maximum of $6 \times 10^{15}$ ions $\mathrm{cm}^{-2}$. Additional samples were irradiated with $150 \mathrm{keV} \mathrm{Kr}^{+}$ions at similar ion fluences using a $200 \mathrm{kV}$ Varian Ion Implanter at the Michigan Ion Beam Laboratory. Plane-view SEM images were taken of the as-irradiated samples using a Phillips XL30 field-emission-gun SEM and an FEI Nova Nanolab Dualbeam FIB operated in SEM mode. Samples were then cleaved, taking care not to damage the delicate surface of the cleaved specimens. Cross-sectional SEM was performed on the cleaved samples using the FEI Nova SEM with an electronbeam incident angle of $90^{\circ}$ to the side of the original sample surface. Composition of the nanofiber layers was confirmed with energy-dispersive X-ray spectroscopy (EDS) analysis using an EDAX UTW detector. Cross-sectional TEM samples were prepared according to standard X-TEM specimen-preparation procedures and observed using a JEOL 2010F analytical electron microscope; EDS was also used to confirm specimen composition using an EDAX r-TEM Detector. The Nova Dualbeam system was also used to perform in situ ion irradiation and SEM. Mirror-polished GaSb samples were irradiated with $30 \mathrm{keV} \mathrm{Ga}^{+}$ions at perpendicular incidence to fluences of up to $6 \times 10^{15}$ ions $\mathrm{cm}^{-2}$; SEM images were taken in situ at an angle of $52^{\circ}$ from the sample surface. The TRIM (Transport and Range of lons in Matter) computer code was used to calculate approximate implant parameters of the $\mathrm{Au}^{+}$ions for use in analyzing observed results. For TRIM calculations, values were defined as: bulk GaSb density of $5.6 \mathrm{~g} \mathrm{~cm}^{-3}$, threshold displacement energy of $25 \mathrm{eV}$ for $\mathrm{Ga}$ and $\mathrm{Sb}$, and surface binding energies of $2.82 \mathrm{eV}$ for $\mathrm{Ga}$ and $2.72 \mathrm{eV}$ for Sb. 


\section{communications}

\section{Keywords:}

embedded nanofibers - ion beams - nanoporous materials . semiconductors

[1] B. R. Bennett, R. Magno, J. B. Boos, W. Kruppa, M. G. Ancona, SolidState Electron. 2005, 49, 1875-1895.

[2] P. S. Dutta, H. L. Bhat, V. Kumar, J. Appl. Phys. 1997, 81, 58215870.

[3] S. Facsko, T. Dekorsy, C. Koerdt, C. Trappe, H. Kurz, A. Vogt, H. L. Hartnagel, Science 1999, 285, 1551-1553.

[4] R. Callec, A. Poudoulec, M. Salvi, H. L'Haridon, P. N. Favennec, M. Gauneau, Nucl. Instrum. Methods Phys. Res. Sect. B 1993, 80/81, 532-537.

[5] C. Schoendorfer, A. Lugstein, E. Bertagnolli, Microelectron. Eng. 2006, 83, 1491-1494.

[6] S. M. Kluth, J. D. Fitz Gerald, M. C. Ridgway, Appl. Phys. Lett. 2005, 86, 131920.

[7] A. Lugstein, C. Schoendorfer, M. Weil, C. Tomastik, A. Jauss, E. Bertagnolli, Nucl. Instrum. Methods Phys. Res. Sect. B 2007, 255, 309-311.

[8] B. R. Appleton, O. W. Holland, J. Narayan, O. E. Schow III, J. S. Williams, K. T. Short, E. Lawson, Appl. Phys. Lett. 1982, 41, 711712.

[9] L. M. Wang, R. C. Birtcher, Appl. Phys. Lett. 1989, 55, 2494-2496.

[10] L. M. Wang, R. C. Birtcher, Philos. Mag. A 1991, 64, 1209-1223.
[11] N. Nitta, M. Taniwaki, Y. Hayashi, T. Yoshiie, J. Appl. Phys. 2002, 92, 1799-1802.

[12] N. Nitta, M. Taniwaki, Nucl. Instrum. Methods Phys. Res. Sect. B 2003, 206, 482-485.

[13] N. Nitta, M. Taniwaki, Physica B 2006, 376/377, 872-876.

[14] N. Nitta, M. Taniwaki, Y. Hayashi, T. Yoshiie, Physica B 2006, 376/ 377, 881-885.

[15] R. Callec, A. Poudoulec, J. Appl. Phys. 1993, 73, 4831-4835.

[16] H. Bracht, S. P. Nicols, W. Walukiewicz, J. P. Silveira, F. Briones, E. E. Haller, Nature 2000, 408, 69-72.

[17] J. F. Ziegler, J. P. Biersack, U. Littmark, The Stopping and Range of Ions in Solids, Pergamon Press, New York 1985. Also see: http:// www.srim.org.

[18] H. Huber, W. Assmann, S. A. Karamian, A. Mücklich, W. Prusseit, E. Gazis, R. Grötzschel, M. Kokkoris, E. Kossionidis, H. D. Mieskes, R. Vlastou, Nucl. Instrum. Methods Phys. Res. Sect. B 1997, 122, 542-546.

[19] E. Le Bourhis, G. Patriarche, Micron 2007, 38, 377-389.

[20] R. Maranganti, P. Sharma, Phys. Rev. Lett. 2007, 98, 195504.

[21] S. K. Mohanta, R. K. Soni, N. Gosvami, S. Tripathy, D. Kanjilal, Appl. Surf. Sci. 2007, 253, 4531-4536.

Received: December 10, 2007 Revised: January 29, 2008 